Proceedings of the Edinburgh Mathematical Society (2007) 50, 363-375 (C)

DOI:10.1017/S0013091505000969 Printed in the United Kingdom

\title{
THE REPRESENTATION OF RESIDUE CLASSES BY PRODUCTS OF SMALL INTEGERS
}

\author{
M. Z. GARAEV ${ }^{1}$ AND A. A. KARATSUBA ${ }^{2}$ \\ ${ }^{1}$ Instituto de Matemáticas, Universidad Nacional Autónoma de México, \\ Campus Morelia, Apartado Postal 61-3 (Xangari), CP 58089, \\ Morelia, Michoacán, Mexico (garaev@matmor.unam.mx) \\ ${ }^{2}$ Steklov Institute of Mathematics, Russian Academy of Sciences, \\ GSP-1, ul. Gubkina 8, Moscow, Russia (karatsuba@mi.ras.ru)
}

(Received 27 June 2005)

\begin{abstract}
For a large integer $m$, we obtain asymptotic formulae for the number of solutions of certain congruences modulo $m$ with several variables, where the variables belong to special sets of residue classes modulo $m$. In particular, we obtain new information on the exceptional set of the multiplication table problem in the residue ring modulo $m$.
\end{abstract}

Keywords: congruences; trigonometric sums; number of solutions; asymptotic formulae

2000 Mathematics subject classification: Primary 11A07; 11B50; 11L07

\section{Introduction}

In this paper we continue to study the distribution properties in residue classes of the sequence consisting of products of two positive integers bounded by a certain parameter.

For a prime number $p$, define the set

$$
\mathcal{A}=\{x y(\bmod p): 1 \leqslant x, y \leqslant N\} .
$$

The main problem is to find a value of $N$, as small as possible, for which any non-zero residue class modulo $p$ would belong to $\mathcal{A}$. The main conjecture is that one can take $N$ to be as small as $p^{1 / 2+o(1)}$.

Vâjâitu and Zaharescu [6] observed that it would completely solve the pair correlation problem for sequences of fractional parts of the form $\left\{\alpha n^{2}\right\}$ (see [5] for the details) if one could deal with the case $N=\left[p^{2 / 3-\varepsilon}\right]$ for some small $\varepsilon>0$. However, it is only known that $N$ can be taken to be of the size $O\left(p^{3 / 4}\right)$ (see $[\mathbf{2}]$ and also $[\mathbf{1}, \mathbf{4}]$ ). The exponent $\frac{3}{4}$ is the best known at the time of writing this paper.

It is shown in [1] that for almost all primes $p$ and $N=\left[p^{1 / 2}(\log p)^{1.087}\right]$ the set $\mathcal{A}$ contains $(1+o(1)) p$ residue classes modulo $p$. It is also conjectured that $\mathcal{A}$ possesses this property for any prime $p$ and $N=\left[p^{1 / 2+\varepsilon}\right]$. We remark that one of our results from $[\mathbf{3}]$ says that for $N=p^{5 / 8+\varepsilon}$ the set $\mathcal{A}$ contains $(1+o(1)) p$ residue classes modulo $p$. 
In this paper we will prove a general statement that in a particular case confirms the validity of the mentioned conjecture from [1] and improves the corresponding result of $[\mathbf{3}]$. The arguments used in $[\mathbf{1}]$ and $[\mathbf{3}]$ are based on estimates of multiplicative character sums. The approach we use here is based on trigonometric sums.

Throughout the text, the letters $p$ and $q$ are used to denote prime numbers, $m$ denotes a positive integer parameter, $S$ and $L$ are some integers with $0<L \leqslant m$. For a given set $\mathcal{Q}$ we use $|\mathcal{Q}|$ to denote its cardinality.

Theorem 1.1. Let $\Delta=\Delta(m) \rightarrow \infty$ as $m \rightarrow \infty$. Then the set

$$
\left\{q y(\bmod m): 1 \leqslant q \leqslant m^{1 / 2}, S+1 \leqslant y \leqslant S+\Delta m^{1 / 2} \sqrt{m / \phi(m)} \log m\right\}
$$

contains $\left(1+O\left(\Delta^{-1}\right)\right) m$ residue classes modulo $m$.

In particular we have the following corollary.

Corollary 1.2. Let $\Delta=\Delta(p) \rightarrow \infty$ as $p \rightarrow \infty$. Then the set

$$
\left\{q y(\bmod p): q \leqslant p^{1 / 2}, 1 \leqslant y \leqslant \Delta p^{1 / 2} \log p\right\}
$$

contains $\left(1+O\left(\Delta^{-1}\right)\right) p$ residue classes modulo $p$.

Since there are $O\left(p^{1 / 2}(\log p)^{-1}\right)$ primes not exceeding $p^{1 / 2}$, we see that the set

$$
\left\{q y: q \leqslant p^{1 / 2}, S+1 \leqslant y \leqslant S+\Delta p^{1 / 2} \log p\right\}
$$

contains only $O(p \Delta)$ integers. This shows that the ranges of variables in Theorem 1.1 and Corollary 1.2 are sharp.

To prove Theorem 1.1, we study the congruence

$$
v_{1}\left(x_{1}+y_{1}\right) \equiv v_{2}\left(x_{2}+y_{2}\right)(\bmod m),
$$

where $v_{1}, v_{2}$ belong to the set of all primes not exceeding $m^{1 / 2}$ and not dividing $m$, and $x_{i}, y_{i}$ run through integers of special intervals. Now we denote by $\mathcal{V}$ any subset of prime numbers not exceeding $m^{1 / 2}$ and not dividing $m$. Let $J$ be the number of solutions of the congruence

$$
v_{1} y_{1} \equiv v_{2} y_{2}(\bmod m), \quad v_{1}, v_{2} \in \mathcal{V}, S+1 \leqslant y_{1}, y_{2} \leqslant S+L .
$$

Theorem 1.3. The following asymptotic formula holds:

$$
J=\frac{|\mathcal{V}|(|\mathcal{V}|-1)}{m} L^{2}+|\mathcal{V}| L+O\left(\frac{m^{2} \log ^{2} m}{\phi(m)}\right),
$$

where $\phi(m)$ is the Euler function.

As we have mentioned, our argument is based on trigonometric sums. In particular, we establish a result on a special trigonometric sum that can be useful in applications to other additive congruences. 
Theorem 1.4. Let $\mathcal{P}$ be any subset of prime numbers not exceeding $p^{1 / 2}$. Then, for any complex coefficients $\alpha_{x}, \beta_{y}$, the formula

$$
\sum_{a=1}^{p-1}\left|\sum_{q \in \mathcal{P}} \sum_{x=1}^{p} \sum_{y=1}^{p} \alpha_{x} \beta_{y} \mathrm{e}^{2 \pi \mathrm{i} a q(x+y) / p}\right|^{2}=|\mathcal{P}| \sum_{a=1}^{p-1}\left|\sum_{x=1}^{p} \sum_{y=1}^{p} \alpha_{x} \beta_{y} \mathrm{e}^{2 \pi \mathrm{i} a(x+y) / p}\right|^{2}+\theta p^{2} I_{1} I_{2}
$$

holds, where $|\theta| \leqslant 1$ and

$$
I_{1}=\sum_{x=1}^{p}\left|\alpha_{x}\right|^{2}, \quad I_{2}=\sum_{y=1}^{p}\left|\beta_{y}\right|^{2}
$$

From Theorem 1.4 one derives the following statement.

Corollary 1.5. Let $\mathcal{X} \subset \mathbb{Z}_{p}, \mathcal{Y} \subset \mathbb{Z}_{p}$, and let $\mathcal{P}$ be any subset of prime numbers not exceeding $p^{1 / 2}$. If $J^{\prime}$ denotes the number of solutions of the congruence

$$
q_{1}\left(x_{1}+y_{1}\right) \equiv q_{2}\left(x_{2}+y_{2}\right)(\bmod p), \quad q_{1}, q_{2} \in \mathcal{P}, x_{1}, x_{2} \in \mathcal{X}, y_{1}, y_{2} \in \mathcal{Y}
$$

then

$$
J^{\prime}=\frac{|\mathcal{P}|(|\mathcal{P}|-1)}{p}|\mathcal{X}|^{2}|\mathcal{Y}|^{2}+|\mathcal{P}| I+\theta p|\mathcal{X}||\mathcal{Y}|,
$$

where $|\theta| \leqslant 1$ and $I$ denotes the number of solutions of the congruence

$$
x_{1}+y_{1} \equiv x_{2}+y_{2}(\bmod p), \quad x_{1}, x_{2} \in \mathcal{X}, y_{1}, y_{2} \in \mathcal{Y} .
$$

Since $I \leqslant|\mathcal{X}|^{3 / 2}|\mathcal{Y}|^{3 / 2}$, we see that if

$$
|\mathcal{P}|^{2}|\mathcal{X}||\mathcal{Y}|=p^{2} \Delta, \quad \Delta=\Delta(p) \rightarrow \infty \text { as } p \rightarrow \infty,
$$

then

$$
J^{\prime}=\frac{|\mathcal{P}|^{2}|\mathcal{X}|^{2}|\mathcal{Y}|^{2}}{p}\left(1+O\left(\Delta^{-1 / 2}\right)\right)
$$

In particular, the set

$$
\{q(x+y)(\bmod p), q \in \mathcal{P}, x \in \mathcal{X}, y \in \mathcal{Y}\}
$$

contains $\left(1+O\left(\Delta^{-1 / 2}\right)\right) p$ residue classes modulo $p$.

Corollary 1.5 also follows from the following statement.

Theorem 1.6. Let $\mathcal{X} \subset \mathbb{Z}_{p}, \mathcal{Y} \subset \mathbb{Z}_{p}$, and let $\mathcal{Z}$ be any subset of positive integers not exceeding $p^{1 / 2}$. If $J^{\prime \prime}$ denotes the number of solutions of the congruence

$$
z_{1}\left(x_{1}+y_{1}\right) \equiv z_{2}\left(x_{2}+y_{2}\right)(\bmod p), \quad z_{1}, z_{2} \in \mathcal{Z}, x_{1}, x_{2} \in \mathcal{X}, y_{1}, y_{2} \in \mathcal{Y}
$$


subject to the additional condition $\left(z_{1}, z_{2}\right)=1$, then

$$
J^{\prime \prime}=\frac{|\mathcal{X}|^{2}|\mathcal{Y}|^{2} T_{\mathcal{Z}}}{p}+\theta p|\mathcal{X}||\mathcal{Y}|
$$

where $|\theta| \leqslant 1$ and $T_{\mathcal{Z}}$ is the number of pairs $z_{1}, z_{2} \in \mathcal{Z}$ with $\left(z_{1}, z_{2}\right)=1$.

We will also prove the following result on the ratio of intervals modulo a prime, which improves one of the results of $[\mathbf{1}]$.

Theorem 1.7. Let $\Delta=\Delta(p) \rightarrow \infty$ as $p \rightarrow \infty$. Then the set

$$
\left\{x y^{-1}(\bmod p): N+1 \leqslant x \leqslant N+\Delta p^{1 / 2}, S+1 \leqslant y \leqslant S+\Delta p^{1 / 2}\right\}
$$

contains $\left(1+O\left(\Delta^{-2}\right)\right) p$ residue classes modulo $p$.

Note, however, that when $N=S=0$ and $\Delta<\frac{1}{2} p^{1 / 2}$ the set described in Theorem 1.7 misses more than $c p^{1 / 2} \Delta^{-1}$ residue classes modulo $p$ for some positive constant $c$ (see [1] ).

The rest of the paper is organized as follows. In $\S 2$ we prove Theorem 1.3. In $\S 3$ we combine the method of $\S 2$ with that described in [2] and establish Theorem 1.1. The rest of the results are proved in $\S \S 4-6$.

In what follows, we use the abbreviation

$$
\boldsymbol{e}_{k}(z)=\mathrm{e}^{2 \pi \mathrm{i} z / k}
$$

\section{Proof of Theorem 1.3}

Recall that $J$ denotes the number of solutions to the congruence

$$
v_{1} y_{1} \equiv v_{2} y_{2}(\bmod m), \quad v_{1}, v_{2} \in \mathcal{V}, S+1 \leqslant y_{1}, y_{2} \leqslant S+L .
$$

We express $J$ in terms of trigonometric sums. Since

$$
v_{1} v_{2}^{-1} y_{1} \equiv y_{2}(\bmod m)
$$

we have

$$
J=\frac{1}{m} \sum_{a=0}^{m-1} \sum_{v_{1} \in \mathcal{V}} \sum_{v_{2} \in \mathcal{V}} \sum_{y_{1} \in \mathcal{I}} \sum_{y_{2} \in \mathcal{I}} \boldsymbol{e}_{m}\left(a\left(v_{1} v_{2}^{-1} y_{1}-y_{2}\right)\right),
$$

where $\mathcal{I}$ denotes the interval $[S+1, S+L]$. Picking up the term corresponding to $a=0$, we obtain

$$
J=\frac{|\mathcal{V}|^{2} L^{2}}{m}+\frac{1}{m} \sum_{a=1}^{m-1} \sum_{v_{1} \in \mathcal{V}} \sum_{v_{2} \in \mathcal{V}} \sum_{y_{1} \in \mathcal{I}} \sum_{y_{2} \in \mathcal{I}} \boldsymbol{e}_{m}\left(a\left(v_{1} v_{2}^{-1} y_{1}-y_{2}\right)\right) .
$$


Furthermore,

$$
\begin{aligned}
& \frac{1}{m} \sum_{a=1}^{m-1} \sum_{v_{1} \in \mathcal{V}} \sum_{v_{2} \in \mathcal{V}} \sum_{y_{1} \in \mathcal{I}} \sum_{y_{2} \in \mathcal{I}} \boldsymbol{e}_{m}\left(a\left(v_{1} v_{2}^{-1} y_{1}-y_{2}\right)\right) \\
& =\frac{1}{m} \sum_{a=1}^{m-1} \sum_{v \in \mathcal{V}} \sum_{y_{1} \in \mathcal{I}} \sum_{y_{2} \in \mathcal{I}} \boldsymbol{e}_{m}\left(a\left(y_{1}-y_{2}\right)\right) \\
& \quad+\frac{1}{m} \sum_{a=1}^{m-1} \sum_{v_{1} \in \mathcal{V}} \sum_{\substack{v_{2} \in \mathcal{V} \\
v_{2} \neq v_{1}}} \sum_{y_{1} \in \mathcal{I}} \sum_{y_{2} \in \mathcal{I}} \boldsymbol{e}_{m}\left(a\left(v_{1} v_{2}^{-1} y_{1}-y_{2}\right)\right) \\
& =|\mathcal{V}| L-\frac{|\mathcal{V}| L^{2}}{m}+\frac{1}{m} \sum_{a=1}^{m-1} \sum_{v_{1} \in \mathcal{V}} \sum_{\substack{v_{2} \in \mathcal{V} \\
v_{2} \neq v_{1}}} \sum_{y_{1} \in \mathcal{I}} \sum_{y_{2} \in \mathcal{I}} \boldsymbol{e}_{m}\left(a\left(v_{1} v_{2}^{-1} y_{1}-y_{2}\right)\right) .
\end{aligned}
$$

Therefore,

$$
J=\frac{|\mathcal{V}|^{2} L^{2}}{m}+|\mathcal{V}| L-\frac{|\mathcal{V}| L^{2}}{m}+\frac{\theta_{1}}{m} \sum_{a=1}^{m-1} \sum_{v_{1} \in \mathcal{V}} \sum_{\substack{v_{2} \in \mathcal{V} \\ v_{2} \neq v_{1}}}\left|\sum_{y_{1} \in \mathcal{I}} \sum_{y_{2} \in \mathcal{I}} \boldsymbol{e}_{m}\left(a\left(v_{1} v_{2}^{-1} y_{1}-y_{2}\right)\right)\right| .
$$

Here and everywhere below, $\theta_{j}$ denotes a function with $\left|\theta_{j}\right| \leqslant 1$.

For a given $n$, let $r(n):=r_{\mathcal{V}}(n)$ be the number of solutions of the congruence

$$
v_{1} v_{2}^{-1} \equiv n(\bmod m), \quad v_{1}, v_{2} \in \mathcal{V}, \quad v_{1} \neq v_{2} .
$$

In particular, $r(1)=0$, and if $(n, m)>1$, then $r(n)=0$. Therefore, the above formula takes the form

$$
J=\frac{|\mathcal{V}|^{2} L^{2}}{m}+|\mathcal{V}| L-\frac{|\mathcal{V}| L^{2}}{m}+\frac{\theta_{1}}{m} \sum_{a=1}^{m-1} \sum_{\substack{1 \leqslant n \leqslant m \\(n, m)=1}} r(n)\left|\sum_{y_{1} \in \mathcal{I}} \sum_{y_{2} \in \mathcal{I}} \boldsymbol{e}_{m}\left(a\left(n y_{1}-y_{2}\right)\right)\right| .
$$

It is important to note that $v^{2} \leqslant m$ for any $v \in \mathcal{V}$. For this reason, we have $r(n) \leqslant 1$ for any $n, 1 \leqslant n \leqslant m$. Indeed, if

$$
v_{1} v_{2}^{-1} \equiv v_{3} v_{4}^{-1}(\bmod m)
$$

for some $v_{1}, v_{2}, v_{3}, v_{4} \in \mathcal{V}$ and if $v_{1} \neq v_{2}$, then

$$
v_{1} v_{4} \equiv v_{3} v_{2}(\bmod m) \text {. }
$$

Since $v^{2} \leqslant m$ for any $v \in \mathcal{V}$, we derive that $v_{1} v_{4}=v_{3} v_{2}$. The elements of $\mathcal{V}$ are prime numbers and $v_{1} \neq v_{2}$. Hence, $v_{1}=v_{3}, v_{2}=v_{4}$.

Thus,

$$
J=\frac{|\mathcal{V}|^{2} L^{2}}{m}+|\mathcal{V}| L-\frac{|\mathcal{V}| L^{2}}{m}+\frac{\theta_{2}}{m} \sum_{a=1}^{m-1} \sum_{\substack{1 \leqslant n \leqslant m \\(n, m)=1}}\left|\sum_{y_{1} \in \mathcal{I}} \sum_{y_{2} \in \mathcal{I}} \boldsymbol{e}_{m}\left(a\left(n y_{1}-y_{2}\right)\right)\right| .
$$


It is now useful to recall the bound

$$
\left|\sum_{y \in \mathcal{I}} \boldsymbol{e}_{m}(b y)\right| \leqslant \frac{1}{|\sin (\pi b / m)|},
$$

which, applied to (2.1), yields

$$
J=\frac{|\mathcal{V}|^{2} L^{2}}{m}+|\mathcal{V}| L-\frac{|\mathcal{V}| L^{2}}{m}+\frac{\theta_{3}}{m} \sum_{a=1}^{m-1} \sum_{\substack{1 \leqslant n \leqslant m \\(n, m)=1}} \frac{1}{|\sin (\pi a n / m)|} \frac{1}{|\sin (\pi a / m)|} .
$$

For each divisor $s \mid m$ we collect together the values of $a$ with $(a, m)=s$. Then

$$
\begin{aligned}
& \sum_{a=1}^{m-1} \sum_{\substack{1 \leqslant n \leqslant m \\
(n, m)=1}} \frac{1}{|\sin (\pi a n / m)|} \frac{1}{|\sin (\pi a / m)|} \\
&=\sum_{\substack{s \mid m \\
s<m}} \sum_{\substack{1 \leqslant a \leqslant m-1 \\
(a, m)=s}} \sum_{\substack{1 \leqslant n \leqslant m \\
(n, m)=1}} \frac{1}{|\sin (\pi a n / m)|} \frac{1}{|\sin (\pi a / m)|} \\
& \leqslant \sum_{\substack{s \mid m \\
s<m}} s \sum_{\substack{1 \leqslant b \leqslant m / s-1 \\
(b, m / s)=1}} \sum_{\substack{1 \leqslant n \leqslant m / s \\
(n, m / s)=1}} \frac{1}{|\sin (\pi b n /(m / s))|} \frac{1}{|\sin (\pi b /(m / s))|} \\
& \leqslant \sum_{\substack{s \mid m \\
s<m}} s\left(\sum_{\substack{1 \leqslant b \leqslant m / s \\
(b, m / s)=1}} \frac{1}{|\sin (\pi b /(m / s))|}\right)^{2} \\
& \ll \sum_{\substack{s \mid m \\
s<m}} s\left(\sum_{1 \leqslant b \leqslant m / 2 s} \frac{m}{b s}\right)^{2} \\
& \leqslant \frac{m^{3} \log ^{2} m}{\phi(m)},
\end{aligned}
$$

where we have used the inequality

$$
\sum_{s \mid m} \frac{1}{s} \leqslant \prod_{p \mid m} \frac{1}{1-p^{-1}}=\frac{m}{\phi(m)} .
$$

Inserting this bound into (2.2), we obtain the required estimate.

\section{Proof of Theorem 1.1}

Without loss of generality, we may assume that

$$
\Delta m^{1 / 2} \sqrt{m / \phi(m)} \log m<m,
$$

as otherwise the statement of Theorem 1.1 is trivial. 
We take $\mathcal{V}$ to be the set of all prime numbers coprime to $m$ and not exceeding $m^{1 / 2}$. Let $J_{1}$ denote the number of solutions to the congruence

$$
v_{1}\left(y_{1}+z_{1}\right) \equiv v_{2}\left(y_{2}+z_{2}\right)(\bmod m)
$$

subject to the conditions

$$
v_{1}, v_{2} \in \mathcal{V}, \quad y_{1}, y_{2}, z_{1}, z_{2} \in \mathcal{I}
$$

where $\mathcal{I}$ denotes the set of integers $x,[S / 2]+1 \leqslant x \leqslant[S / 2]+L$, and

$$
L=\left[\frac{\Delta m^{1 / 2} \sqrt{m / \phi(m)} \log m}{2}\right] .
$$

It is obvious that

$$
S+1 \leqslant y_{i}+z_{i} \leqslant S+\Delta m^{1 / 2} \sqrt{m / \phi(m)} \log m, \quad i=1,2 .
$$

Following the lines of the proof of Theorem 1.3, we express $J_{1}$ in terms of trigonometric sums. Since

$$
v_{1} v_{2}^{-1}\left(y_{1}+z_{1}\right) \equiv y_{2}+z_{2}(\bmod m)
$$

we have

$$
J_{1}=\frac{1}{m} \sum_{a=0}^{m-1} \sum_{v_{1} \in \mathcal{V}} \sum_{v_{2} \in \mathcal{V}} \sum_{y_{1}, z_{1} \in \mathcal{I}} \sum_{y_{2}, z_{2} \in \mathcal{I}} \boldsymbol{e}_{m}\left(a\left(v_{1} v_{2}^{-1}\left(y_{1}+z_{1}\right)-y_{2}-z_{2}\right)\right) .
$$

Picking up the term corresponding to $a=0$, we obtain

$$
J_{1}=\frac{|\mathcal{V}|^{2} L^{4}}{m}+\frac{1}{m} \sum_{a=1}^{m-1} \sum_{v_{1} \in \mathcal{V}} \sum_{v_{2} \in \mathcal{V}} \sum_{y_{1}, z_{1} \in \mathcal{I}} \sum_{y_{2}, z_{2} \in \mathcal{I}} \boldsymbol{e}_{m}\left(a\left(v_{1} v_{2}^{-1}\left(y_{1}+z_{1}\right)-y_{2}-z_{2}\right)\right) .
$$

Since the number of solutions of the congruence

$$
y_{1}+z_{1} \equiv y_{2}+z_{2}(\bmod m), \quad y_{1}, z_{1}, y_{2}, z_{2} \in \mathcal{I},
$$

is $O\left(L^{3}\right)$, we obtain

$$
\frac{1}{m}\left|\sum_{a=1}^{m-1} \sum_{v \in \mathcal{V}} \sum_{y_{1}, z_{1} \in \mathcal{I}} \sum_{y_{2}, z_{2} \in \mathcal{I}} \boldsymbol{e}_{m}\left(a\left(y_{1}+z_{1}-y_{2}-z_{2}\right)\right)\right| \leqslant \frac{|\mathcal{V}|}{m} \sum_{a=0}^{m-1}\left|\sum_{y \in \mathcal{I}} \boldsymbol{e}_{m}\left(a y_{1}\right)\right|^{4} \ll|\mathcal{V}| L^{3}
$$

Therefore,

$$
\begin{aligned}
& \frac{1}{m} \sum_{a=1}^{m-1} \sum_{v_{1} \in \mathcal{V}} \sum_{v_{2} \in \mathcal{V}} \sum_{y_{1}, z_{1} \in \mathcal{I}} \sum_{y_{2}, z_{2} \in \mathcal{I}} \boldsymbol{e}_{m}\left(a\left(v_{1} v_{2}^{-1}\left(y_{1}+z_{1}\right)-y_{2}-z_{2}\right)\right) \\
& =O\left(|\mathcal{V}| L^{3}\right)+\frac{1}{m} \sum_{a=1}^{m-1} \sum_{v_{1} \in \mathcal{V}} \sum_{\substack{v_{2} \in \mathcal{V} \\
v_{2} \neq v_{1}}} \sum_{y_{1} \in \mathcal{I}} \sum_{y_{2} \in \mathcal{I}} \boldsymbol{e}_{m}\left(a\left(v_{1} v_{2}^{-1}\left(y_{1}+z_{1}\right)-y_{2}-z_{2}\right)\right) .
\end{aligned}
$$


Using exactly the same argument that we used in the proof of Theorem 1.3, we derive the formula

$$
J_{1}=\frac{|\mathcal{V}|^{2} L^{4}}{m}+O\left(|\mathcal{V}| L^{3}\right)+O(R)
$$

where

$$
R=\frac{1}{m} \sum_{a=1}^{m-1} \sum_{\substack{1 \leqslant n \leqslant m \\(n, m)=1}}\left|\sum_{y_{1}, z_{1} \in \mathcal{I}} \sum_{y_{2}, z_{2} \in \mathcal{I}} \boldsymbol{e}_{m}\left(a\left(n\left(y_{1}+z_{1}\right)-y_{2}-z_{2}\right)\right)\right| .
$$

Next, introducing $s=(a, m)$, we obtain

$$
\begin{aligned}
R & =\frac{1}{m} \sum_{\substack{s \mid m \\
s<m}} \sum_{\substack{b \leqslant m / s-1 \\
(b, m / s)=1}} \sum_{\substack{1 \leqslant n \leqslant m \\
(n, m)=1}}\left|\sum_{y_{1}, z_{1} \in \mathcal{I}} \sum_{y_{2}, z_{2} \in \mathcal{I}} \boldsymbol{e}_{m / s}\left(b\left(n\left(y_{1}+z_{1}\right)-y_{2}-z_{2}\right)\right)\right| \\
& \leqslant \frac{1}{m} \sum_{\substack{s \mid m \\
s<m}} s \sum_{\substack{b \leqslant m / s-1 \\
(b, m / s)=1}} \sum_{\substack{1 \leqslant n \leqslant m / s \\
(n, m / s)=1}}\left|\sum_{y_{1}, z_{1} \in \mathcal{I}} \sum_{y_{2}, z_{2} \in \mathcal{I}} \boldsymbol{e}_{m / s}\left(b n\left(y_{1}+z_{1}\right)-b\left(y_{2}+z_{2}\right)\right)\right| \\
& \leqslant \frac{1}{m} \sum_{\substack{s \mid m \\
s<m}} s\left(\sum_{\substack{1 \leqslant n \leqslant m / s \\
(n, m / s)=1}}\left|\sum_{y_{1}, z_{1} \in \mathcal{I}} \boldsymbol{e}_{m / s}\left(n\left(y_{1}+z_{1}\right)\right)\right|\right)^{2} \\
& =\frac{1}{m} \sum_{\substack{s \mid m \\
s<m}} s\left(\sum_{\substack{1 \leqslant n \leqslant m / s \\
(n, m / s)=1}}\left|\sum_{y \in \mathcal{I}} \boldsymbol{e}_{m / s}(n y)\right|^{2}\right)^{2}
\end{aligned}
$$

Therefore,

$$
J_{1}=\frac{|\mathcal{V}|^{2} L^{4}}{m}+O\left(|\mathcal{V}| L^{3}\right)+O\left(R_{1}\right)+O\left(R_{2}\right)
$$

where

$$
\begin{aligned}
& R_{1}=\frac{1}{m} \sum_{\substack{s \mid m \\
s<m / L}} s\left(\sum_{\substack{1 \leqslant n \leqslant m / s \\
(n, m / s)=1}}\left|\sum_{y \in \mathcal{I}} \boldsymbol{e}_{m / s}(n y)\right|^{2}\right)^{2}, \\
& R_{2}=\frac{1}{m} \sum_{\substack{s \mid m \\
m / L \leqslant s<m}} s\left(\sum_{\substack{1 \leqslant n \leqslant m / s \\
(n, m / s)=1}}\left|\sum_{y \in \mathcal{I}} \boldsymbol{e}_{m / s}(n y)\right|^{2}\right)^{2} .
\end{aligned}
$$

If $s<m / L$, then $m / s>L$ and, therefore, the congruence

$$
y_{1} \equiv y_{2}(\bmod m / s), \quad y_{1}, y_{2} \in \mathcal{I},
$$

has $L$ solutions. Hence,

$$
\sum_{1 \leqslant n \leqslant m / s}\left|\sum_{y \in \mathcal{I}} \boldsymbol{e}_{m / s}(n y)\right|^{2}=\frac{m L}{s}
$$


whence, using (3.2),

$$
\begin{aligned}
R_{1} & \leqslant \frac{1}{m} \sum_{\substack{s \mid m \\
s<m / L}} s\left(\sum_{1 \leqslant n \leqslant m / s}\left|\sum_{y \in \mathcal{I}} \boldsymbol{e}_{m / s}(n y)\right|^{2}\right)^{2} \\
& =m L^{2} \sum_{\substack{s \mid m \\
s<m / L}} s^{-1} \\
& \leqslant m L^{2} \sum_{s \mid m} s^{-1} \\
& \leqslant \frac{m^{2} L^{2}}{\phi(m)} .
\end{aligned}
$$

Inserting this bound into (3.1), we deduce that

$$
J=\frac{|\mathcal{V}|^{2} L^{4}}{m}+O\left(|\mathcal{V}| L^{3}\right)+O\left(m^{2} L^{2} / \phi(m)\right)+O\left(R_{2}\right) .
$$

We now proceed to estimate $R_{2}$. Note that in $(3.3)$ we have $(n, m / s)=1$. Therefore, for any integer $K$,

$$
\sum_{y=K+1}^{K+m / s} \boldsymbol{e}_{m / s}(n y)=0,
$$

whence we deduce that there exist integers $A$ and $B$ with $0<B \leqslant m / s$ such that

$$
\sum_{y \in \mathcal{I}} \boldsymbol{e}_{m / s}(n y)=\sum_{A<y \leqslant A+B} \boldsymbol{e}_{m / s}(n y)
$$

Hence

$$
\begin{aligned}
\sum_{\substack{1 \leqslant n \leqslant m / s \\
(n, m / s)=1}}\left|\sum_{y \in \mathcal{I}} \boldsymbol{e}_{m / s}(n y)\right|^{2} & =\sum_{\substack{1 \leqslant n \leqslant m / s \\
(n, m / s)=1}}\left|\sum_{A<y \leqslant A+B} \boldsymbol{e}_{m / s}(n y)\right|^{2} \\
& \leqslant \sum_{n=1}^{m / s}\left|\sum_{A<y \leqslant A+B} \boldsymbol{e}_{m / s}(n y)\right|^{2} \\
& =m B / s \leqslant m^{2} / s^{2} .
\end{aligned}
$$

Taking this into account, from (3.3) we deduce that

$$
R_{2} \leqslant \frac{1}{m} \sum_{s \geqslant m / L} s\left(m^{4} / s^{4}\right) \ll m L^{2}
$$

Therefore, in view of (3.4), we obtain the asymptotic formula

$$
\begin{aligned}
J_{1} & =\frac{|\mathcal{V}|^{2} L^{4}}{m}+O\left(|\mathcal{V}| L^{3}\right)+O\left(m^{2} L^{2} / \phi(m)\right) \\
& =\frac{|\mathcal{V}|^{2} L^{4}}{m}\left(1+O\left(\frac{m}{|\mathcal{V}| L}+\frac{m^{3}}{\phi(m)|\mathcal{V}|^{2} L^{2}}\right)\right) .
\end{aligned}
$$


Recalling that $|\mathcal{V}| \gg m^{1 / 2} / \log m$ and

$$
L=\left[\frac{\Delta m^{1 / 2} \sqrt{m / \phi(m)} \log m}{2}\right]
$$

we arrive at the formula

$$
J_{1}=\frac{|\mathcal{V}|^{2} L^{4}}{m}\left(1+O\left(\Delta^{-1}\right)\right)
$$

Next, define

$$
\mathcal{H}=\{q(y+z)(\bmod m), q \in \mathcal{V},[S / 2]+1 \leqslant y, z \leqslant[S / 2]+L\} .
$$

Obviously, $S+1 \leqslant y+z \leqslant S+\Delta m^{1 / 2} \sqrt{m / \phi(m)} \log m$. For a given $h \in \mathcal{H}$, by $I(h)$ we denote the number of solutions of the congruence

$$
q(y+z) \equiv h(\bmod m), \quad q \in \mathcal{V},[S / 2]+1 \leqslant y, z \leqslant[S / 2]+L .
$$

Then

$$
J_{1}=\sum_{h \in \mathcal{H}} I^{2}(h) \geqslant \frac{1}{|\mathcal{H}|}\left(\sum_{h \in \mathcal{H}} I(h)\right)^{2}=\frac{1}{|\mathcal{H}|}|\mathcal{V}|^{2} L^{4}
$$

Therefore,

$$
|\mathcal{H}| \geqslant \frac{|\mathcal{V}|^{2} L^{4}}{J_{1}}=\frac{m}{1+O\left(\Delta^{-1}\right)}=\left(1+O\left(\Delta^{-1}\right)\right) m .
$$

The result now follows in view of $|\mathcal{H}| \leqslant m$.

\section{Proof of Theorem 1.4}

Set

$$
S=\sum_{a=1}^{p-1}\left|\sum_{q \in \mathcal{P}} \sum_{x=1}^{p} \sum_{y=1}^{p} \alpha_{x} \beta_{y} \boldsymbol{e}_{p}(a q(x+y))\right|^{2}
$$

In the identity

$$
\sum_{a=1}^{p-1} \boldsymbol{e}_{p}(a u)= \begin{cases}-1, & \text { if } u \neq \equiv(\bmod p), \\ p-1, & \text { if } u \equiv 0(\bmod p)\end{cases}
$$

we successively take $u=q_{1}\left(x_{1}+y_{1}\right)-q_{2}\left(x_{2}+y_{2}\right)$ and then

$$
u=q_{1} q_{2}^{-1}\left(x_{1}+y_{1}\right)-\left(x_{2}+y_{2}\right),
$$

where $q_{2}^{-1}$ is defined from $q_{2} q_{2}^{-1} \equiv 1(\bmod p)$, and obtain

$$
\sum_{a=1}^{p-1} \boldsymbol{e}_{p}\left(a\left(q_{1}\left(x_{1}+y_{1}\right)-q_{2}\left(x_{2}+y_{2}\right)\right)\right)=\sum_{a=1}^{p-1} \boldsymbol{e}_{p}\left(a\left(q_{1} q_{2}^{-1}\left(x_{1}+y_{1}\right)-\left(x_{2}+y_{2}\right)\right)\right) .
$$

Multiplying both sides by $\alpha_{x_{1}} \bar{\alpha}_{x_{2}} \beta_{y_{1}} \bar{\beta}_{y_{2}}$, performing the summation over

$$
q_{1}, q_{2} \in \mathcal{P}, \quad 1 \leqslant x_{1}, x_{2}, y_{1}, y_{2} \leqslant p,
$$


and then changing the order of summation, we obtain

$$
S=\sum_{a=1}^{p-1} \sum_{\substack{q_{1} \in \mathcal{P} \\ q_{2} \in \mathcal{P}}} \sum_{\substack{x_{1} \in \mathbb{Z}_{p} \\ x_{2} \in \mathbb{Z}_{p}}} \sum_{\substack{y_{1} \in \mathbb{Z}_{p} \\ y_{2} \in \mathbb{Z}_{p}}} \alpha_{x_{1}} \bar{\alpha}_{x_{2}} \beta_{y_{1}} \bar{\beta}_{y_{2}} \boldsymbol{e}_{p}\left(a q_{1} q_{2}^{-1}\left(x_{1}+y_{1}\right)-a\left(x_{2}+y_{2}\right)\right),
$$

where $\mathbb{Z}_{p}=\{1,2 \ldots, p\}$. The contribution to $S$ which comes from the case $q_{1}=q_{2}$ is equal to

$$
\begin{aligned}
|\mathcal{P}| \sum_{a=1}^{p-1} \sum_{\substack{x_{1} \in \mathbb{Z}_{p} \\
x_{2} \in \mathbb{Z}_{p}}} \sum_{y_{1} \in \mathbb{Z}_{p}} \alpha_{x_{1}} \overline{\mathbb{Z}}_{p} \bar{\alpha}_{x_{2}} \beta_{y_{1}} \bar{\beta}_{y_{2}} \boldsymbol{e}_{p}\left(a\left(x_{1}+y_{1}-x_{2}-y_{2}\right)\right) & \\
& =|\mathcal{P}| \sum_{a=1}^{p-1}\left|\sum_{x=1}^{p} \sum_{y=1}^{p} \alpha_{x} \beta_{y} \boldsymbol{e}_{p}(a(x+y))\right|^{2} .
\end{aligned}
$$

Therefore,

$$
S=|\mathcal{P}| \sum_{a=1}^{p-1}\left|\sum_{x=1}^{p} \sum_{y=1}^{p} \alpha_{x} \beta_{y} \boldsymbol{e}_{p}(a(x+y))\right|^{2}+S_{1},
$$

where

$$
S_{1}=\sum_{a=1}^{p-1} \sum_{\substack{q_{1} \in \mathcal{P} \\ q_{2} \in \mathcal{P} \\ q_{1} \neq q_{2}}} \sum_{\substack{x_{1} \in \mathbb{Z}_{p} \\ x_{2} \in \mathbb{Z}_{p}}} \sum_{y_{1} \in \mathbb{Z}_{p}} \alpha_{y_{2} \in \mathbb{Z}_{p}} \bar{\alpha}_{x_{2}} \beta_{y_{1}} \bar{\beta}_{y_{2}} \boldsymbol{e}_{p}\left(a q_{1} q_{2}^{-1}\left(x_{1}+y_{1}\right)-a\left(x_{2}+y_{2}\right)\right) .
$$

Hence, if we prove that $\left|S_{1}\right| \leqslant p^{2} I_{1} I_{2}$, then we are done. To this end, we observe that

$$
\left|S_{1}\right| \leqslant \sum_{a=1}^{p-1} \sum_{n=1}^{p-1} r(n)\left|\sum_{\substack{x_{1} \in \mathbb{Z}_{p} \\ x_{2} \in \mathbb{Z}_{p}}} \sum_{\substack{y_{1} \in \mathbb{Z}_{p} \\ y_{2} \in \mathbb{Z}_{p}}} \alpha_{x_{1}} \bar{\alpha}_{x_{2}} \beta_{y_{1}} \bar{\beta}_{y_{2}} \boldsymbol{e}_{p}\left(a n\left(x_{1}+y_{1}\right)-a\left(x_{2}+y_{2}\right)\right)\right|,
$$

where $r(n):=r_{\mathcal{P}}(n)$ denotes the number of solutions of the representation

$$
q_{1} q_{2}^{-1} \equiv n(\bmod p), \quad q_{1}, q_{2} \in \mathcal{P}, \quad q_{1} \neq q_{2} .
$$

From the definition of the set $\mathcal{P}$ we derive that $r(n) \leqslant 1$. Hence,

$$
\left|S_{1}\right| \leqslant \sum_{a=1}^{p-1} \sum_{n=1}^{p-1}\left|\sum_{\substack{x_{1} \in \mathbb{Z}_{p} \\ x_{2} \in \mathbb{Z}_{p}}} \sum_{\substack{y_{1} \in \mathbb{Z}_{p} \\ y_{2} \in \mathbb{Z}_{p}}} \alpha_{x_{1}} \bar{\alpha}_{x_{2}} \beta_{y_{1}} \bar{\beta}_{y_{2}} \boldsymbol{e}_{p}\left(\operatorname{an}\left(x_{1}+y_{1}\right)-a\left(x_{2}+y_{2}\right)\right)\right| .
$$

When $n$ runs through the reduced residue system modulo $p$, an runs through the same system for any fixed $a \not \equiv 0(\bmod p)$. Therefore,

$$
\begin{aligned}
\left|S_{1}\right| & \leqslant\left(\sum_{a=1}^{p-1}\left|\sum_{x=1}^{p} \sum_{y=1}^{p} \alpha_{x} \beta_{y} \boldsymbol{e}_{p}(a(x+y))\right|\right)^{2} \\
& =\left(\sum_{a=1}^{p-1}\left|\sum_{x=1}^{p} \alpha_{x} \boldsymbol{e}_{p}(a x)\right|\left|\sum_{y=1}^{p} \beta_{y} \boldsymbol{e}_{p}(a y)\right|\right)^{2} .
\end{aligned}
$$


Applying the Cauchy inequality, we obtain

$$
\left|S_{1}\right| \leqslant\left(\sum_{a=0}^{p-1}\left|\sum_{x=1}^{p} \alpha_{x} \boldsymbol{e}_{p}(a x)\right|^{2}\right)\left(\sum_{a=0}^{p-1}\left|\sum_{y=1}^{p} \beta_{y} \boldsymbol{e}_{p}(a y)\right|^{2}\right)=p^{2} I_{1} I_{2},
$$

which concludes our proof of Theorem 1.4.

\section{Proof of Theorem 1.6}

The proof proceeds along exactly the same lines as that of Theorem 1.4: by remarking that, for any given residue class $n$, the congruence

$$
z_{1} z_{2}^{-1} \equiv n(\bmod p), \quad z_{1}, z_{2} \in \mathcal{Z},\left(z_{1}, z_{2}\right)=1,
$$

has at most one solution.

\section{Proof of Theorem $\mathbf{1 . 7}$}

Without loss of generality we may suppose that

$$
0<N<N+\Delta p^{1 / 2}<p, \quad 0<M<M+\Delta p^{1 / 2}<p .
$$

Define $X=\left[\Delta p^{1 / 2} / 2\right], N_{1}=[N / 2], S_{1}=[S / 2]$, and let $\mathcal{H}^{*}$ be the set of all residue classes of the form $(x+t)(y+z)^{-1}(\bmod p)$, where

$$
N_{1}+1 \leqslant x, t \leqslant N_{1}+X, \quad S_{1}+1 \leqslant y, z \leqslant S_{1}+X .
$$

Obviously,

$$
N+1 \leqslant x+t \leqslant N+\Delta p^{1 / 2}, \quad S+1 \leqslant y+z \leqslant S+\Delta p^{1 / 2} .
$$

Next, let

$$
\mathcal{H}_{1}^{*}=\left\{h(\bmod p): h \notin \mathcal{H}^{*}, h \not \equiv 0(\bmod p)\right\} .
$$

Then the congruence

$$
x+t-(y+z) h \equiv 0(\bmod p)
$$

has no solutions in variables $h, x, t, y, z$ subject to the conditions

$$
h \in \mathcal{H}_{1}^{*}, \quad N_{1}+1 \leqslant x, t \leqslant N_{1}+X, \quad S_{1}+1 \leqslant y, z \leqslant S_{1}+X .
$$

Therefore,

$$
\sum_{a=0}^{p-1} \sum_{h \in \mathcal{H}_{1}^{*}} \sum_{x, t \in \mathcal{I}_{1}} \sum_{y, z \in \mathcal{I}_{2}} e_{p}(a(x+t-h(y+z)))=0,
$$

where $\mathcal{I}_{1}$ and $\mathcal{I}_{2}$ denote the intervals $\left[N_{1}+1, N_{1}+X\right]$ and $\left[S_{1}+1, S_{1}+X\right]$, respectively. 
Separating the term corresponding to $a=0$ we deduce that

$$
\left|\mathcal{H}_{1}^{*}\right| X^{4} \leqslant \sum_{a=1}^{p-1}\left|\sum_{x, t \in \mathcal{I}_{1}} \boldsymbol{e}_{p}(a(x+t))\right|\left|\sum_{y, z \in \mathcal{I}_{2}} \sum_{h \in \mathcal{H}_{1}^{*}} \boldsymbol{e}_{p}(a h(y+z))\right| .
$$

On the other hand, for $(a, p)=1$, we have

$$
\begin{aligned}
\left|\sum_{y, z \in \mathcal{I}_{2}} \sum_{h \in \mathcal{H}_{1}^{*}} \boldsymbol{e}_{p}(a h(y+z))\right| & \leqslant \sum_{h \in \mathcal{H}_{1}^{*}}\left|\sum_{y, z \in \mathcal{I}_{2}} \boldsymbol{e}_{p}(a h(y+z))\right| \\
& \leqslant \sum_{h=1}^{p-1}\left|\sum_{y, z \in \mathcal{I}_{2}} \boldsymbol{e}_{p}(a h(y+z))\right| \\
& \leqslant \sum_{h=0}^{p-1}\left|\sum_{y, z \in \mathcal{I}_{2}} \boldsymbol{e}_{p}(h(y+z))\right| \\
& =p X
\end{aligned}
$$

and, similarly,

$$
\sum_{a=1}^{p-1}\left|\sum_{x, t \in \mathcal{I}_{1}} e_{p}(a(x+t))\right| \leqslant p X .
$$

Hence,

$$
\left|\mathcal{H}_{1}^{*}\right| X^{4} \leqslant p^{2} X^{2},
$$

whence

$$
\left|\mathcal{H}_{1}^{*}\right| \leqslant \frac{p^{2}}{X^{2}} \ll p \Delta^{-2} .
$$

Since $|\mathcal{H}|=p-1-\left|\mathcal{H}_{1}^{*}\right|$, the result follows.

Acknowledgements. The authors are highly thankful to the anonymous referee for many useful comments.

\section{References}

1. M. Z. GARAEv, Character sums in short intervals and the multiplication table modulo a prime, Monatsh. Math. 148 (2006), 127-138.

2. M. Z. GARAEv, On the logarithmic factor in error term estimates in certain additive congruence problems, Acta. Arith. 124 (2006), 27-39.

3. M. Z. Garaev and A. A. Karatsuba, On character sums and the exceptional set of a congruence problem, J. Number Theory 114 (2005), 182-192.

4. M. Z. Garaev And K.-L. KueH, Distribution of special sequences modulo a large prime, Int. J. Math. Math. Sci. 50 (2003), 3189-3194.

5. Z. Rudnik, P. SARnak And A. Zaharescu, The distribution of spacings between the fractional parts of $n^{2} \alpha$, Invent. Math. 145(1) (2001), 37-57.

6. M. VÂJÂITU AND A. ZAhARESCU, Differences between powers of a primitive root, Int. J. Math. Math. Sci. 29(2) (2002), 325-331. 